\title{
HOSPITAL CAPACITY PLANNING FOR EFFICIENT DISASTER MITIGATION DURING A BIOTERRORIST ATTACK
}

\author{
Jomon Aliyas Paul \\ Govind Hariharan \\ 1000 Chastain Road \\ MD 0403, BB, Bldg. 4, Rm. 322 \\ Michael J. Coles College of Business, Kennesaw State University \\ Kennesaw, GA 30144-5591, U.S.A.
}

\begin{abstract}
Effective hospital capacity planning can not only significantly enhance the capability and effectiveness of the treatment provided to patients during a bioterrorist attack but can also provide critical information. While a lot of work has been done to model hospital capacity estimates for natural disasters the same cannot be said for manmade biological disasters like anthrax or smallpox. In this paper, we develop a generic simulation model of hospital capacity planning during a bioterrorist attack. We model both cases in which the occurrence of the attack and the type of agent used are known as well as when they are not known. The model is also unique in developing a feedback loop to alert emergency management officials about the occurrence and type of an attack. Our results are able to pinpoint the characteristics of the hospitals that are most relevant at various stages of exposure and provide policy recommendations.
\end{abstract}

\section{INTRODUCTION}

Man-made disasters, such as terrorist attack, industrial accidents, acts of war, can result in significant economic loss and human casualties. One of the most recent and significant man-made disasters occurred on September 11, 2001, when the World Trade Center in New York City was attacked by terrorists. Approximately 2750 people were killed and another 2260 injured. The economic losses suffered have been in the order of trillions of dollars (Wikipedia $2001_{\mathrm{a}}$ ). This was followed in September and October of 2001 by a bioterrorist attack involving anthrax in which around 22 people became ill and resulted in 5 deaths (Wikipedia 2001 ).

The effective functioning of a medical facility is critical during an emergency. Unlike a natural disaster like earthquake or floods, bioterrorist attacks entail a different set of complexities with respect to care delivery. Firstly, it might not be possible to instantly identify the biological agents based on the symptoms that patients come into a hospital with. For example, the early symptoms of smallpox are akin to chickenpox while that of anthrax to any common bacterial infection. It often remains to the lab tests to be able to determine the true ailment and this might take time. Secondly, patients suffering from contagious diseases such as smallpox may need to be quarantined from other patients. Thirdly, the resources required such as the time of nurses and doctors will depend on the agent A nurse or doctor has to change the personal protective equipment (PPE) every time they attend to a patient thereby consuming a lot of time just getting ready to treat a patient. Fourthly, unlike a natural disaster patients affected by these bioterrorism agents may not need the services of operating rooms (OR). This changes the constraints on a hospital with respect to number of surgical units/surgical services. Finally, facility damage to the hospital itself are less likely to exist in the event of such bioterrorist attacks.

All of this points to the fact that any simulation modeling of hospitals for bioterrorist attacks will have to take into account a number of factors which are very unique and specific to these disaster types. In this paper, we develop a generic simulation model of hospital capacity planning during a bioterrorist attack that takes into account these factors. We model both cases in which the occurrence of the attack and the type of agent used are known as well as when they are not known. We also characterize the differences between contagious and noncontagious diseases. The model is unique in developing a feedback loop to alert emergency management officials about the occurrence and type of an attack. Our results are able to pinpoint the characteristics of the hospitals that are most relevant at various stages of exposure and provide policy recommendations.

\section{LITERATURE REVIEW}

The most relevant literature for this study are models of hospital capacity estimation. McClure (1976) described 


\section{Paul and Hariharan}

hospital capacity to be the combination of bed and intensity care capacities. HEW (1977) projected bed requirements five years ahead by (1) using population, (2) applying current per-capita hospital experience to obtain expected utilization, and (3) applying an ideal occupancy factor to obtain beds required. Roemer and Shain (1959) concluded that beds beget patients, in the sense that beds will ultimately be occupied at approximately the same rate in any hospital even if bed number is increased. Bailey projected the need for future beds for both certified and non-certified beds in a three-year planning horizon for the US counties (Bailey 1994). Trye et al. (2002) constructed a quantitative mathematical model for estimating future bed demand based on two years of seasonally adjusted inpatient data. Mouza (2002) projected hospital bed requirements by the end of the planning period based on the consistent forecasts of the admission rates taking into account the structure of the admitted population by sex and age groups.

Some models have been developed that take into account other important factors besides beds and therefore, are more robust. Boer et al. 1989 defined hospital treatment capacity (HTC) as the number of casualties that can be treated according to normal medical standards in one hour. It depends on the total number of surgeons, anesthesiologists, operating rooms, intensive care beds, residents, and the like. Yi et al. 2006 have developed a generic simulation model for real time estimation of hospital capacities during a bioterrorist attack disaster situation. Patvivatsiri (2006) developed a simulation model of emergency room to better prepare for a bioterrorist attack. The objective of the model was to identify the bottleneck resources and determine their appropriate allocation levels to better handle sudden surge of patients resulting from a bioterrorist attack.

\section{METHODOLOGY}

The agents that could be used in a bioterrorist attack can lead to two types of diseases: noncontagious but still deadly like anthrax or contagious like smallpox with equally (or even) higher deadly effects. In this paper, we specifically focus on developing a generic hospital model for anthrax but discuss the implications for smallpox. The important aspects to be considered for the simulation model are discussed in the sections below.

\subsection{Characteristics of Bioterrorist Attacks}

In this section we describe the differing characteristics of anthrax and smallpox which make mitigation very different and much more complicated than natural disasters.

\subsubsection{Symptoms}

There are three possible forms of the disease anthrax, the most common of which is cutaneous anthrax. in which the organism enters through a break in the skin. The cutaneous form begins as a bump that looks like an insect bite and within days opens into a painless ulcer with a black area in the center. The patient may have fever, malaise and headache. Mortality of cutaneous anthrax victims ranges from $20-25 \%$ without treatment, less than one percent with treatment. A more serious form is inhalation anthrax in which the victim breathes in the organism and develops a severe respiratory disease. The signs and symptoms of inhalational anthrax follow a two stage pattern. In the first stage, the symptoms commonly observed are viral respiratory illness, sore throat, mild fever, muscle aches, malaise etc. In the second and fulminant stage, the symptoms observed are shortness of breath, fever, shock, meningitis, respiratory failure etc. (Defencejournal 98, Tasota etal. 2002, Labtestonline 2006). Systemic infection resulting from inhalation of Bacillus anthracis has a mortality rate approaching $100 \%$. The third form is gastrointestinal anthrax which occurs 2 to 5 days after the ingestion of undercooked infected meat.

Smallpox is a highly contagious and virulent, often fatal infectious disease caused by variola virus, a large orthopoxvirus of the family Poxviridae, subfamily Chordopoxvirinae (afip 2006). There are four types of smallpox that a person could get a)Classical variety b)Hemarroghic c)Malignant d)Modified. The symptoms develop in two stages. First stage symptoms primarily include aching pains, prostration, rash, lesions, bleeding into the skin and intestinal tract etc. The second stage symptoms include pneumonia, meningal irritation etc. Malignant smallpox is the most severe one and modified is the least variety of smallpox.

\subsubsection{Contamination}

During a bioterrorist attack, it is normal for many patients to present themselves as contaminated victims (AHRQ 2004). For faster disaster mitigation it is critical that these patients be decontaminated. There are three main purposes for carrying out this procedure: 1) make victim's skin and clothing free of the agent in order to reduce further possible agent exposure and further effects among victims; 2) to protect the emergency responders and other hospital staff from secondary transfer exposures, which is the primary rationale for ensuring detailed decontamination at the health care facility, and 3) to provide psychological comfort to victims at or near the incident site to mitigate negative long-term psychological impact (Tan 2003). 


\section{Paul and Hariharan}

\subsubsection{Quarantine}

For some infectious diseases like smallpox and SARS which spreads at an alarming rate from an infected person, it might be necessary to totally quarantine a hospital or a region treating such cases to avoid any further contamination. A hospital might have to be totally closed for normal patients while treating the infected ones. An alternative is for the hospital to be kept open to normal patients with the infected or contaminated ones treated at offsite locations. These might require extensive equipment and staffing to be effective. They also impose a constraint on the available capacity and thereby the number of people that could be given necessary care.

\subsubsection{Lab capacity and Tests}

Laboratories play a critical role in the response to bioterrorist attack, as the timeliness, accuracy, and security of lab diagnostics will have a direct impact on the containment and mitigation of an incident and on the effectiveness of treatment to victims.

\subsection{Characteristics of Hospitals For Simulation Modeling}

The hospitals of interest during a bioterrorist attack are those that treat general conditions like fever, GI problems, nausea etc. Specialty hospitals such as cancer institutes, psychiatric centers, etc., are not seen as significant contributors to the treatment of bioterrorist attack related conditions. Only non-specialty hospitals are thus included in this study.

We classify hospitals according to their characteristics, e.g., by size, location, etc. The capacity of a hospital is associated with the number of beds as well as its care delivery system (PAHO 1995), where the latter is essentially its surgical capabilities. The casualties resulting from a bioterrorist attack rarely need surgical services however, a large percentage of patients require ventilator support in addition to other care, and thereby the role of intensive care units becomes very critical during bioterrorist attacks.

\subsubsection{Number of beds}

After studying more than 50 hospitals randomly selected from different states across the country and data from AHA (Health Forum 2001-2002), we consider a typical large hospital to have about 500 beds, a medium-sized one to have 300 beds, and a small one with about 100 beds. We therefore categorize hospitals into three sizes with 100 , 300 , and 500 beds. Hospitals within the range can be interpolated from the obtained results. Lab capacity has been shown to be related to the number of beds (Yi et al 2006).
The higher the number of beds in a hospital the higher is the capacity of the lab in that hospital. The functional relationship between Lab capacity and number of beds is as follows:

$\mathrm{L}_{\mathrm{CAP}}=\operatorname{ROUND}\left((92 * \mathrm{~B}+2267) / \mathrm{LCAP} \_\mathrm{Scaler}+3\right)$

where $\mathrm{L}_{\mathrm{CAP}}$ is Lab capacity, $\mathrm{B}$ is the number of beds and $\mathrm{L}_{\mathrm{CAP} \_ \text {scaler }}$ is a constant that depends on the size of the hospital.

\subsubsection{Number of Intensive Care Units (ICU)}

Intensive care units play a critical role since patients subject to anthrax (especially cutaneous and inhalational anthrax) or smallpox generally suffer from severe respiratory distress and require the services of ventilation and critical care (Rubinson L. et al. 2005). Thus, the number of intensive care units in a hospital would have a significant impact on the capacity available at the hospital to treat such patients and we include it as factor in our model. Intensive care unit is a specialized facility that provides intensive care medicine. Many hospitals also have designated intensive care areas for certain specialties of medicine as dictated by the needs and available resources of each hospital.

Based on the data collected from around 150 hospitals across United States, we estimated the number of ICUs in hospitals to range from 4 through 104 (AHD 2006). Small hospitals with around 100 beds have approximately 5 ICUs, a medium sized hospital with around 300 beds have 15 and large hospitals with around 500 beds have 35 ICUs.

\subsubsection{Intensive Care Unit Efficiency}

Even for hospitals with the same number of beds and ICUs, the number of patients treated varies widely with various degrees of ICU efficiency. Performance of ICU in terms of patient mortality has been shown to be related to patient length of stay in ICU (BJS 2002 and Bandolier 2003). Lesser the number of vent days the more successful are the outcomes. Thus, a logical measure of ICU performance is the patient length of stay.

We obtained data for patient length of stay in ICU by patient type (Cerner APACHE 2007). The mean length of stay by patient type was found to be not statistically different by patient type or diagnosis. We used this data to create a confidence interval for length of stay. The lower limit, overall mean and upper limit of this confidence interval are used as estimates for low, medium and high efficiency ICUs. The ICU efficiency values we have considered in this paper are 4, 4.5 and 5 respectively .

\subsubsection{Severity Separation and Priority Assignment}

Patient separation based on severity and priority assignment based on severity has been shown to be a more realistic modeling of hospital operations (Saunders et al. 1989). 


\section{Paul and Hariharan}

In this paper, we consider three different severities. Patients are given priorities based on these three severity types. Each patient is assigned a survivability time corresponding to his/her severity. The survivability time is the maximum time that a patient can wait before getting treatment in ER or ICU or else he/she dies. Severity 3 will always get priority over 2 and 1 . Similarly severity 2 will get priority over 1 .

\subsubsection{Change in Patient Mix}

Patient mix has been identified as an important factor affecting the available resources and thereby, waiting time of the patients. Studies have shown that clinical environments are highly sensitive to small changes in patient mix and patient scheduling rules. The performance of the ICUs has been noticed to be significantly different if they have different case mixes (Glance et al 2000). The standardized mortality ratio was found to be different for ICU's dealing with different case mixes.

In this paper we classify patient mix to be of three types, one: ratio of Severity 1 patients/Total number of patients denoted by $\alpha$, two: ratio of Severity $2 /$ Total number of patients denoted by $\beta$, and three: Number of severity $3 /$ Total number of patients denoted by $\gamma$. The effect of the change in these factors on the patient waiting times will be studied by running the model for different values of $\alpha, \beta$ and $\gamma$.

\subsection{Design of Simulation Experiments}

The four factors (beds, ICU, ICU efficiency, and patient mix) are considered in the design of a factorial experiment to obtain a set of experimental results, which will used in the subsequent regression. We have not considered infeasible combinations like a hospital with around 100 beds with 15 ICUs or 35 ICUs. These narrow it down to only 15 feasible combinations as shown in Figure 1 .

\section{SIMULATION MODEL OF HOSPITALS}

\subsection{Generic Simulation Model of Hospitals}

Using the simulation software ProModel, we developed a generic hospital model using the partial factorial design described earlier. In a disaster situation, all staff will be called on duty. Due to the same reasons, human resource and equipment constraints are not considered in this phase of the study.

Since capacity estimate cannot be directly measured from the simulation outputs, we need to identify the relevant indicators. Although utilization shows the available capacity of a resource, this long-term performance measure does not relate directly to the number of additional patients the hospital can treat. Since it is possible to treat an increased influx of patients if prolonged waiting times are allowed, the capacity estimate also depends on the allowable waiting time. Therefore, actual waiting times are a meaningful measure. Additionally, waiting can be related to capacity subject to the survivability time (maximum waiting time a patient can wait before the treatment begins). In order to do so, we consider the waiting time to be the time a patient waits for treatment to begin.

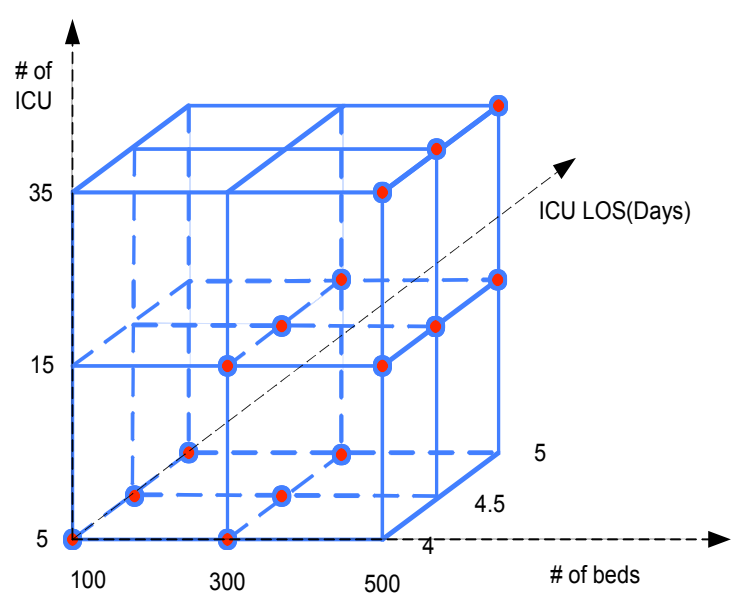

Figure 1: Experimental design factors and levels(Dots indicate the 15 feasible combinations we have considered for our study)

\subsection{Patient Types}

Based on the similarity of medical symptoms and treatment procedures, patients were categorized into three types of Anthrax patients:

Type 1 patients: bumps on skin, sore throat (Respiratory), mild fever (Any system), muscle aches (Any system), sever cramps, malaise (Any system - general symptoms, swelling in the mouth or esophagus, swollen lymph nodes.

Type 2 Patients: shortness of breath (Respiratory), fever (Any system), meningitis (Neuro), respiratory failure (Respiratory), viral respiratory illness (Respiratory), severe nausea (Gastrointestinal), loss of appetite, vomiting (Gastrointestinal or Neuro), vomiting blood (GI or Neuro), bloody diarrhea (GI), delirium, sweating, severe difficulty breathing

Type 3 Patients: severe abdominal stress (Cardiovascular), abdominal pain (Cardio).

Based on patient data available from the 2001 anthrax episodes, we assume that patients have an average incubation period of 4 days after attack, prodormal stage setting in 4 days after incubation before going into the fulminant phase (Jernigan et. al. 2001). This logic is used in separating the patients into different severity levels for the attack known-cause known scenario that we plan to simulate. In 


\section{Paul and Hariharan}

addition, in the attack and cause known case, it is expected that there will be higher numbers of severity 1 patients compared to attack known-cause unknown and attack unknown-cause unknown cases. The percentages of patients by type are obtained from historical data (Holty et al 2006). Patient routings after anthrax attacks can be seen in Figure 3.

\subsection{Modeling Logic}

Unlike natural disasters like earthquakes it may not be immediately apparent that a bioterrorist attack has oc curred. Even if it were known that an attack has occurred, the type of agent and whether it is contagious or not may not be known immediately. The biological attack can entail different scenarios. Either the moment attack occurs the time might be known and the cause might be known or either may be known or only one may be known. This might lead to different care delivery needs in the hospital. To our knowledge this is the first model to capture this critical component of biological disasters. A visualization of the same is provided in the Figure 2.

If the occurrence of attack is known and the type of agent is known the patient is assigned to the ER bed or ICU/CCU depending on his stability as shown in Figure 6. Similarly if the occurrence of the attack is not known and type of agent is not known, the actual cause of the problems the patients comes in with is known only when lab results are known.

Once the patient arrives at the hospital he will follow the flowchart in Figure 3. One unique aspect of our model is the feedback loop that alerts public health officials to the possibility that an attack has occurred and the type of agent involved. The biological agent triage algorithm we utilized is the one previously developed by Bond et al. 2006.

We begin by studying the attack known and cause known case first. We analyze the effect of different patient volumes and severities on hospital capacity estimates for both contagious and non contagious diseases

\section{CAPACITY PREDICTION MODEL}

\subsection{Pre-disaster Steady-state Waiting Time Equation}

For each of the different hospital settings in the factorial design, we obtain the steady-state pre-disaster waiting time from simulation. Then, a metamodel in regression of these results relates pre-disaster steady-state waiting time to number of beds, number of ICUs, and efficiency:

$\mathrm{T}=\mathrm{C}_{0}+\mathrm{C}_{1} \mathrm{~B}+\mathrm{C}_{2} \mathrm{I}+\mathrm{C}_{3} \mathrm{E}+\mathrm{C}_{4} \mathrm{~A}+\mathrm{C}_{5} \mathrm{~B}^{2}+\mathrm{C}_{6} \mathrm{I}^{2}+\mathrm{C}_{7} \mathrm{E}^{2}+$ $\mathrm{C}_{8} \mathrm{~A}^{2}+\mathrm{C}_{9} \mathrm{BI}+\mathrm{C}_{10} \mathrm{BE}+\mathrm{C}_{11} \mathrm{BA}+\mathrm{C}_{12} \mathrm{IE}+\mathrm{C}_{13} \mathrm{IA}+\mathrm{C}_{14} \mathrm{EA}$ where $\mathrm{T}$ is the steady-state waiting time under normal times, B is number of beds in the hospital, I is number of ICU's, E is efficiency index, $\mathrm{A}$ is patient mix (representing all the three $\alpha, \beta, \gamma)$ and $\mathrm{C}_{0}, \mathrm{C}_{1}, \ldots$ are constants.

\subsection{Post-disaster Steady-state Waiting Time Equation}

Post-disaster waiting time regression equations have the same form as the pre-disaster waiting time equation. The patient volumes after the bioterrorist attack that can be treated may vary from zero volume of patients to a threshold maximum volume.

\subsubsection{Post-disaster Base and Critical Cases}

If after the bioterrorist attack, no disaster patients arrive, there would only be a minimum arrival rate of regular ER patients, resulting in a large capacity available. This patient volume is a hypothetical base case. When any disaster takes place the incoming patient volume would be greater than or equal to this base case. Using the same regression approach, we obtain the steady-state base case waiting time for any given hospital.

By experimenting on various post-disaster arrival rates, the critical arrival rate will be determined. That is, any sustained rate greater than this critical rate would push the system into in equilibrium. We call this situation the critical case, where the system is on the threshold of becoming over- capacitated.

\subsubsection{Arrival Rates between the Base Case and the Critical Case}

In order to find the relationship between patient arrival rate and the steady-state waiting time under situations between the base case and the critical case, we will perform a series of simulations with different arrival rates for each hospital. Waiting time increases exponentially with patient arrival rate. There is a good log-linear relationship between waiting time and patient arrival rate. Therefore, for any given hospital, the following relationship holds:

$$
\log \left(\mathrm{T}_{s}\right)=a+b * \lambda
$$

where $a$ and $b$ are constants, $\mathrm{T}_{s}=$ Steady-state waiting time and $\lambda=$ Patient arrival rate

For any given hospital combination, the above equation corresponds to a straight line in a two-dimensional space of Log (Steady-state waiting time) and Patient arrival rate. Therefore, the constants $a$ and $b$ can be uniquely determined by two points known to lie on the line. These points correspond to the base case and the critical case. It is important to note that $a$ and $b$ depend on four basic hospital factors only, which strongly supports our research 


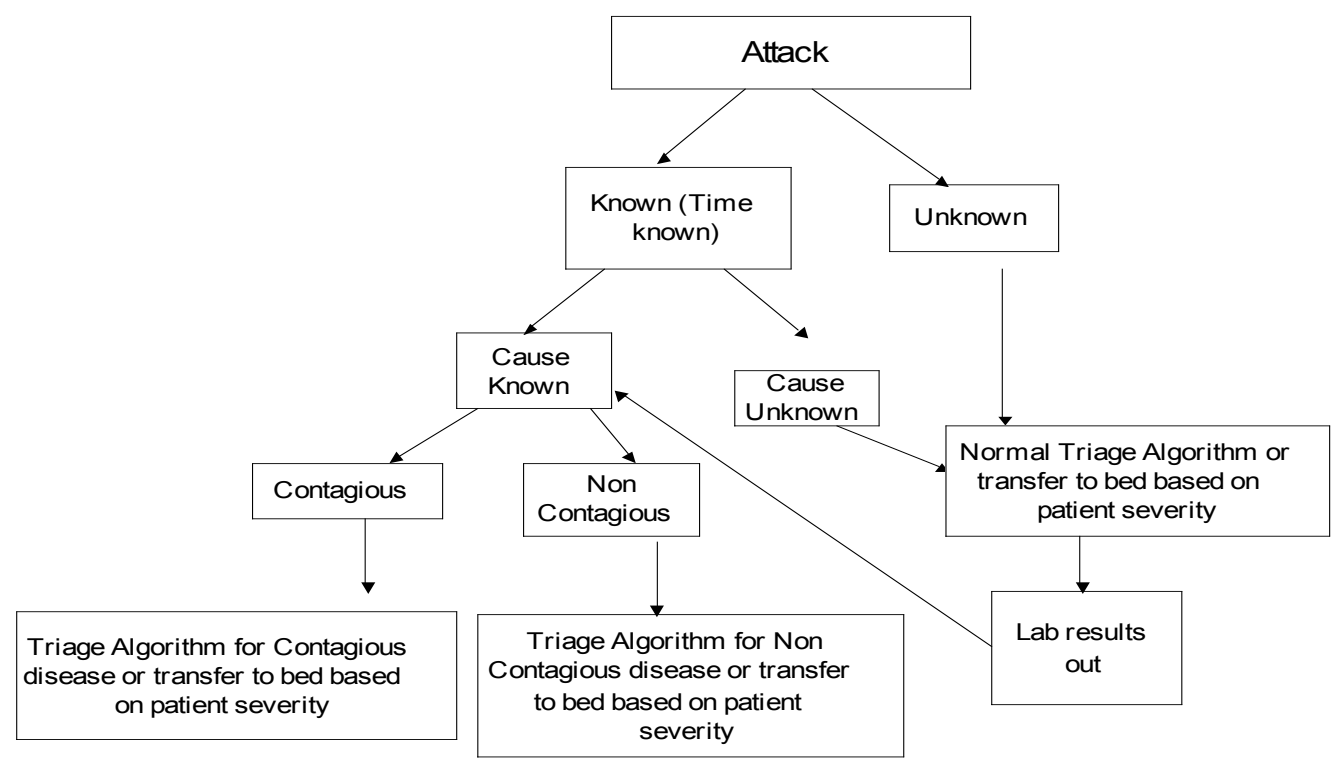

Figure 2: Flow chart (Attack Scenarios)
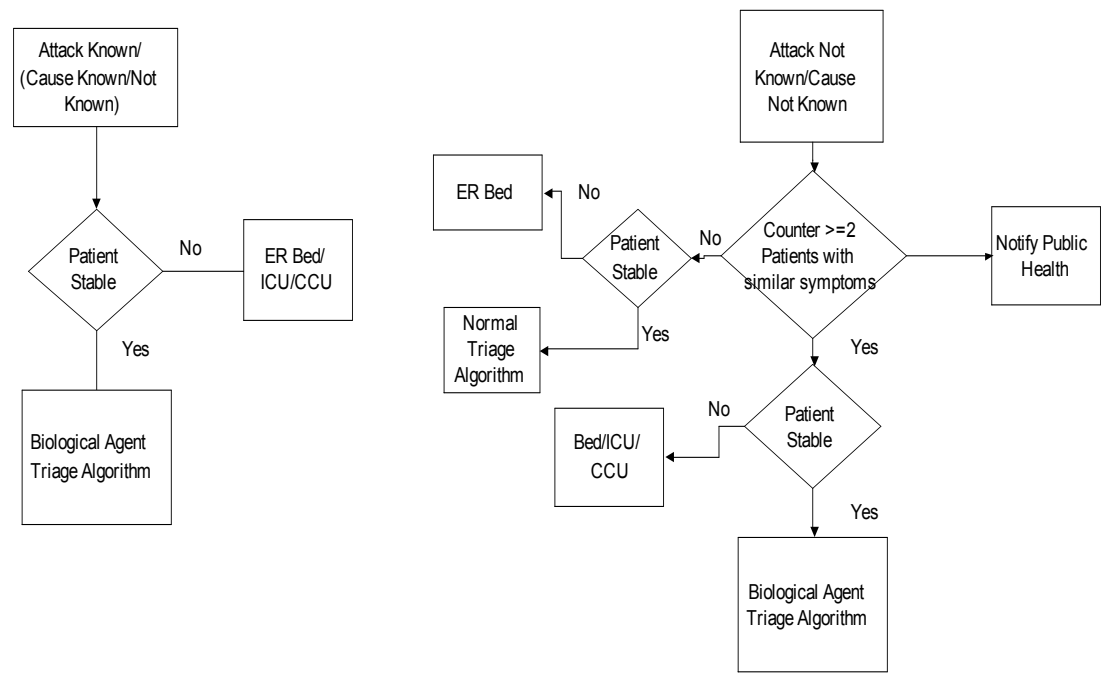

Figure 3: Flow chart (Patient Flow)

objective on developing generic hospital models to represent all hospitals in the disaster area.

\subsection{Transient State Waiting Times}

The hospital transient behavior can be described by the weighted sum of two exponential functions. The two exponential functions take into account all arrival rates from the base case to the critical case and any state in between. The time of bioterrorist attack is $t_{e q}$; the transient waiting time at clock time $t$ is $T_{r}(t)$; the steady state waiting time before bioterrorist attack is $T_{i}$; and the steady-state waiting time corresponding to the patient arrival rate after the bioterrorist attack is $T_{f}$. Assuming static but different pre-disaster and post-disaster arrival rates, the following equation is obtained,

$$
\begin{aligned}
T_{r}(t) & =\mathrm{T}_{i}+(1-\alpha) \cdot\left(\mathrm{T}_{f}-\mathrm{T}_{i}\right) \cdot\left(1-e^{\left(\frac{t_{t_{a}}-t}{\tau_{1}}\right)}\right) \\
& +\alpha \cdot\left(\mathrm{T}_{f}-\mathrm{T}_{i}\right) \cdot\left(1-e^{\left(\frac{t_{c a}-t}{\tau_{2}}\right)}\right)
\end{aligned}
$$

For dynamic post-disaster arrival rates, the relation can be generalized as follows. If at time $t_{l}$ the waiting time is $T_{l}$, the patient arrival rate $\lambda$ during the transient (within a time interval from $t_{1}$ to $t_{2}$ ) is a constant, and the steady- 


\section{Paul and Hariharan}

state waiting time corresponding to $\lambda$ is $T_{2}$, then the transient waiting time $T_{r}(t)$ is given by

$$
\begin{gathered}
T_{r}(t)=T_{1}+(1-\alpha)\left(T_{2}-T_{1}\right)\left(1-e^{\frac{t_{1}-t}{\tau_{1}}}\right) \\
+\alpha\left(T_{2}-T_{1}\right)\left(1-e^{\frac{t_{1}-t}{\tau_{2}}}\right)
\end{gathered}
$$

where $\tau_{1}$ and $\tau_{2}$ are two time constants corresponding to the base case and critical case, respectively. They are approximately linearly related to the time it will take to reach from a pre-disaster steady state to the post-disaster steady state. The higher the value of $\tau$, the longer it will take to reach a new steady state from the previous steady state and vice-versa. Weighting factor $\alpha$ is between 0 and 1 . In the base case, $\alpha=0$, and in the critical case, $\alpha=1$. The value of $\alpha$ can be obtained by performing several simulations with different post-disaster patient arrival rates for selected hospital combinations.

\subsection{Capacity Estimation}

Since our ultimate objective is to estimate hospital capacity in terms of the number of patients that the hospital can accept with an acceptable waiting time not exceeding survivability time, it is necessary to convert the waiting times into capacity estimates.

Assuming the maximum permitted waiting time is $T_{m}$, from equation (2), in steady state, this waiting time for a maximum patient arrival rate $\left(\lambda_{\mathrm{m}}\right)$ given by

$$
\lambda_{m}=\left(\ln \left(T_{m}\right)-a\right) / b
$$

Assuming the current waiting time (transient waiting time from double exponential curve) to be a steadystate waiting time for a certain patient arrival rate, this arrival rate $\left(\lambda_{\mathrm{s}}\right)$ can be calculated as

$$
\lambda_{s}=\ln \left(T_{r}(t)-a\right) / b
$$

where $T_{r}(t)$ is current waiting time.

Then the available capacity is equal to the difference between the maximum capacity and the used capacity

$$
C=\left(\lambda_{m}-\lambda_{s}\right) \cdot \Delta t
$$

where $\Delta \mathrm{t}$ is length of time and $C$ is available capacity.

If the length of time is one hour, then the available hourly capacity $\left(C_{h}\right)$ is

$$
C_{h}=\lambda_{m}-\lambda_{s}
$$

\section{RESULTS}

\subsubsection{Results for Attack and Cause Known Scenario (Anthrax).}

For incubation stage patients (Severity 1) the results are:

$\mathrm{T}=C_{0}+C_{1} B+C_{2} V+C_{3} B^{2}+C_{4} V^{2}+C_{5} B V$

R-square $=94.9 \%$

C_vol $=C_{0}+C_{1} B+C_{2} B^{2}, \quad$ R-square $=97 \%$, where $C_{-}$vol is the critical volume that the hospital can handle.

Similarly for prodormal stage patients (Severity 2) $\mathrm{T}=C_{0}+C_{1} I+C_{2} E+C_{3} V+C_{4} I^{2}+C_{5} E^{2}+C_{6} V^{2}+C_{7} B I+$ $C_{8} B E+C_{9} B V+C_{10} I E+C_{11} I V+C_{12} E V$, R-square=97.6\%

C vol $=\mathrm{C}_{0}+\mathrm{C}_{1} \mathrm{I}+\mathrm{C}_{2} \mathrm{E}+\mathrm{C}_{3} \mathrm{I}^{2}+\mathrm{C}_{4} \mathrm{E}^{2}+\mathrm{C}_{5} \mathrm{BI}+\mathrm{C}_{6} \mathrm{BE}+$ $\overline{\mathrm{C}_{7} \mathrm{IE}}, \quad \mathrm{R}$-square $=98 \%$

and finally for fulminant Stage Patients (Severity 3)

$\mathrm{T}=C_{0}+C_{1} I+C_{2} E+C_{3} V+C_{4} I^{2}+C_{5} E^{2}+C_{6} V^{2}+C_{7} B I+$ $C_{8} B E+C_{9} B V+C_{10} I E+C_{11} I V+C_{12} E V$, R-square $=98.4 \%$

C_vol $=C_{0}+C_{1} I+C_{2} E+C_{3} I^{2}+C_{4} E^{2}+C_{5} B I+C_{6} B E+$ $C_{7} I E, \quad$ R-square $=98.7 \%$

\subsubsection{Implications for Attack Known and Cause Unknown Scenario}

Some states such as Georgia do not permit hospitals to conduct testing of potentially infected patients in their own labs while some other states do. The results developed earlier readily apply to the latter but not to the former. In states that do not allow such testing, hospitals are required to send samples to the CDC.. In these states, the waiting time and critical volume results will remain the same, however mortality rates will be significantly higher. This is because hospitals will have to wait for results from CDC which generally takes a time period of 1-3 days (CDC 2007). This delays the appropriate care delivery to patients and thereby increase the mortality. It is likely to be not so severe in a non contagious disease like anthrax but extremely serious in the case of contagious diseases like smallpox where patients can infect others including health care workers and pose a big problem for achieving efficient disaster mitigation (provide reference).

\subsection{Empirical Observations}

The stage at which patients enter the hospital has a huge impact on which hospital characteristics matter most. 


\section{Paul and Hariharan}

1) Incubation patients are not affected by the ICU number or ICU efficiency of a hospital but are affected by the number of beds that a hospital has (ER and labs) and the volume it takes. This is because they do not need the services of ICU. They require only the care delivery given to a normal ER patient. 2) Prodormal and incubation patients are not affected significantly by the number of beds that a hospital has. The major bottleneck for these patients is the number of ICUs, ICU efficiency and patient volume. Beds do not show up as a significant factor because the hospital gets bogged down due to the very low ratio of ICU/Beds that any hospital has. 3) The critical volume of patients that a hospital can bear is highest for incubation, the second are prodormal and the lowest are fulminant patients. This is mainly a function of the severity of these three categories of patients and the type of care that each of the three categories need. 4) Policies banning testing in some states should be changed. All states must permit hospitals to do testing in their labs since it has a significant impact on mortality. In addition, the turnaround of the results is faster, more accurate and less risk prone than when sent to the CDC (RID 2007).

\section{CONCLUSIONS AND FUTURE WORK}

In this paper, we developed a generic hospital simulation model to obtain temporal performance measures (waiting time) during emergency operations for anthrax attacks. We model cases in which the occurrence of the attack is known and the type of agent used are known as well as cases in which they are not known. We have highlighted the differences between contagious and noncontagious diseases. We developed a parametric regression metamodel to obtain real-time hospital capacity estimates from simulation results. The model is also unique in developing a feedback loop to alert emergency management officials about the occurrence and type of an attack. Our model is also able to point out those characteristics of hospitals that are of critical importance at various stages.

We have not simulated the scenario where both attack and cause of agent are not known in this paper. In addition due to the lack of smallpox patient data, we have simulated only anthrax attack. We have also not considered different patient mixes to see its impact on the waiting times and hospital critical volumes. This would be a very important factor especially in the attack and cause unknown scenario. In addition bottleneck that is created due to shortage of nurses and doctors also needs to be modeled. These are all important aspects that will be pursued in our future work.

\section{ACKNOWLEDGMENTS}

We would like to thank the anonymous referees, Dr. Monte Masten, Dr. Andrew Kramer, Dr. Tom Kim, Dr. Marcia
Delk and Wellstar Institute For Better Health for their invaluable comments.

\section{REFERENCES}

AFIP 2006.

<http://www.afip.org/Departments/inf ectious/sp/txt/1_1.htm>.

AHD 2006. <http://www . ahd.com/>.

AHRQ 2004. Optimizing surge capacity: regional efforts in bioterrorism readiness. Bioterrorism and Health System Preparedness, Issue Brief No. 4. AHRQ Publication No. 04-P009.

Bailey J. C. 1994. Long term bed need projections. Indiana State Department of Health Information Services Commission, Health Planning Division, Indianapolis, IN.

Bandolier 2003. Physician staffing and ICU outcomes. <http://www.jr2.ox.ac.uk/bandolier/b and107/b107-4.html>.

BJS 2002. Intensivists reduce mortality, length of stay in ICU. <http://ww.scienceblog.com/cms/ node/318/print>.

Boer J de, Brismar B., Eldar R., and Rutherford W. H. 1989. The medical severity index of disasters. The Journal of Emergency Medicine; 7(3): 269-273.

Bond W. F., Subbarao I., Schwid H. A., Bair A. E., and Johnson C. 2006. Using screen-based computer simulation to develop and test a civilian, symptom-based terrorism triage algorithm. International Trauma Care;16: 19-25.

HEW, Bureau of Health Planning. Health care facilities existing and needed; Hill-Burton State Plan Data as of Jan. 1975.

CDC

2007.

<http://www.bt.cdc.gov/agent/anthrax /fag/labtesting.asp>

Cerner APACHE 2006. ICU length of stay data for hospitals in United States by diagnosis.

Defencejournal 1998. Anthrax as a weapon of terrorism and difficulties presented in response to its use. <www.defencejournal.com/dec98/anthra $\mathrm{x} \cdot \mathrm{htm}>$

Glance L. G., Osler T., and Shinozaki T. 2000. Effect of varying the case mix on the standardized mortality ratio and W statistic: a simulation study. Chest; 117:1112-1117.

Gruenberg D. A., Shelton W., Rose S. L., Rutter A. E., Socaris S. and McGee G. 2006. Factors influencing length of stay in the intensive care unit. American Journal of Critical Care; 15(5): 502-509.

Health Forum 2001-2002. AHA guide to the healthcare field, edition, American Hospital Association, Chicago, IL. 


\section{Paul and Hariharan}

Henderson D. A., Inglesby T. V., and Bartlett J. G. 1999. Smallpox as a biological weapon: medical and public health management. JAMA 281:2127-2137.

HEW, Bureau of Health Planning 1977. Health care facilities existing and needed; Hill-Burton State Plan Data as of January 1975, Washington, DC..

Holty J. C., Bravata D. M., Liu H., Olshen R. A., McDonald K. M., and Owens D. K. 2006. Systematic Review: A century of inhalational anthrax cases from 1900 to 2005. Annals of Internal Medicine 144:270-280.

Kahn J. M., Goss C. H., Heagerty P. J., Kramer A. A., o'Brien, Chelsea R., and Rubenfeld G. D. 2006. Hospital volume and the outcomes of mechanical ventilation. The New England Journal of Medicine 355:4150 .

Jackson B. A., Baker J. C., Ridgely M. S., Bartis J. T., and Linn H. I. 2004. Safety management in disaster and terrorism response. Protecting Emergency Responders, Rand Science and Technology Institute and National Institute of Occupational Safety and Health; 3:MG-170.

Jernigan J.A. et al. 2001. Bioterrorism-related inhalational anthrax: the first 10 cases reported in the United States. Emerging Infectious Diseases 7(6): 933-944.

Labtestonline 2006. <http://www.labtestonline.org/unders tanding/conditions/anthrax.html>

McClure W. 1976. Reducing excess hospital capacity. U.S. Department of Commerce.

Mouza A. M. 2002. Estimation of the total number of hospital admissions and bed requirements for 2011: the case for Greece. Health services management research 15(3): 186-192.

Patvivatsiri L. 2006. Simulation model for bioterrorism preparedness in an emergency room. In Proceedings of the 2006 Winter Simulation Conference 501-508.

RID 2007. Newly released data confirm CDC failures and the urgent need for cleanliness standards and MRSA screening in U.S. hospitals.

Roemer M., and Shain M. 1959. Hospital utilization under insurance. American Hospital Association, Chicago, IL.

Rubinson L., Nuzzo, J. B., Talmor, D. S. , O'Toole T. Kramer, B. R. , Inglesby, T. V. 2005. Augmentation of hospital critical care capacity after bioterrorist attacks or epidemics: recommendations of the working group on emergency mass critical care, Critical Care Medicine; 33(10): E1-E13.

Saunders C. E., Makens P. K., and Leblanc L. J. 1989. Modeling emergency department operations using advanced computer simulation systems. Annals of Emergency Medicine 18(2):134-140.

Tan, G.A. and M.C. Fitzgerald. 2003. Chemical-biologicalradiological (CBR) response: a template for hospital emergency departments. Medical Journal of Australia 178(3): 141.

Tasota F. J., Henker R. A., and Hoffman L. A. 2002. Anthrax as a biological weapon: An old disease that poses a new threat. Critical Care Nurse; 21-36.

Trye P., Murray N., Wolstencroft I., and Stewart A. 2002. Health service capacity modeling. Australian health review: a publication of the Australian Hospital Association; 25(4):159-168.

Wikipedia 2001a. September 11, 2001 attacks; <http://en.wikipedia.org/wiki/Septem ber_11,2001_attacks>.

Wikipedia 2001b. 2001 anthrax attacks; <http://en.wikipedia.org/wiki/2001_a nthrax_attacks>.

Wikipedia 2006 <http://en.wikipedia.org/wiki/Intens ive care unit>.

Yi P., George S. A., Paul J. A., and L. Lin. 2006. Hospital capacity planning for emergency management in disaster mitigation. SocioEconomic Planning Sciences (in print).

Zeng X., and Wagner M. 2006. Modeling of patient treatment seeking behavior after bioterrorism attack: rationale for data sources integration and simulation parameters selection. Center for Biomedical Informatics, University of Pittsburgh, Pittsburgh, PA15260.

\section{AUTHOR BIOGRAPHIES}

JOMON ALIYAS PAUL is an Assistant Professor at the Coles College of Business, Kennesaw State University. His research focus includes application of Operations research and GIS in disaster management, statistical modeling and simulation modeling of hospitals and airport security systems. His current research focuses on planning for manmade disasters. He is an ASQ certified Six Sigma black belt.

GOVIND HARIHARAN is Chair and Professor at the Coles College of Business, Kennesaw State University. He specializes in Health Economics, the Economics of Regulations, and Financial Economics. He has published many seminal papers and has been cited extensively in scientific and government publications including the Regulatory Program of the United States and the Surgeon General's report on Smoking and Health. task forces. Dr. Hariharan serves on the Board of the Wellstar Institute, the Leadership Council of the American Diabetes Association (Atlanta Chapter) and is a member of many health related task forces. 